\title{
Optimization of Kinetic Energy Harvester for Low Amplitude Vibration
}

\author{
Brian Dick, Mark Fralick, Hugo Jazo, Maxwell Kerber, Jerry Brewer, Richard Waters (Senior Member IEEE) \\ Advanced Circuits and Sensors Branch \\ Space and Naval Warfare System Center Pacific (SSC Pacific) \\ San Diego, CA, USA \\ richard.waters@,navy.mil
}

\begin{abstract}
This paper presents the steps involved in optimizing the design of an electromagnetic kinetic energy harvester (KEH). The KEH device is conceptually a highly non-linear device. There are numerous dependent variables involved in the design of a KEH which are reliant upon the specific environmental conditions in which the KEH will be deployed. Furthermore, the non-linear nature of the device leads to an iterative design process. The environment that the KEH is deployed into also dictates the overall design and power per volume achieved by the device.
\end{abstract}

\section{INTRODUCTION}

The use of kinetic energy harvesters $(\mathrm{KEH})$ to extract energy from the environment provides a continuous source of power that can be used for wireless sensors. Energy harvesters reduce the cost of replacing batteries and hard wiring, and also mitigate the required entry into hazardous or hard to access locations. There are various energy harvester transduction methodologies that have been developed and that are commercially available including solar, thermoelectric, and kinetic [1]. Solar energy harvesting is perhaps the most common and most widely recognized form of energy harvesting but it has limited application in many wireless sensor networks (WSN) due to low light levels found in many application environments Thermoelectric transducers suffer from very low efficiencies for low temperature gradients making them impractical for many desired WSN application environments. The focus of this paper will be to look into the construction of kinetic energy harvesters. Specifically, we will examine the construction of a novel electro-magnetic energy transducer to harvest kinetic energy. This paper will focus on the design of these $\mathrm{KEH}$ for low amplitude vibrations that are typical in many environments.

Vibration energy harvesters are devices that are continually being researched and developed, however, they are mostly made to operate at $0.2 \mathrm{~g}$ (where $1 \mathrm{~g}=9.81 \mathrm{~m} / \mathrm{s}^{2}$ ) and higher [2], [3]. However, Space and Naval Warfare System Center Pacific (SSC Pacific), along with others [4], have found that there are a lot of environments that provide vibration accelerations much less than $100 \mathrm{mg}$. This limits the use of these $\mathrm{KEH}$ to areas with either continuous high accelerations or to periodic operating time. Because there is a desire for continuously powered devices, such as sensors, $\mathrm{KEH}$ need to be diversified for all environments. To meet this need SSC PACIFIC has developed KEH that use a patented

U.S. Government work not protected by U.S. copyright 1840 design [5], which operate on accelerations of $100 \mathrm{mg}$ peak and less.

The patented design consists of two rigidly attached magnets, with like poles facing, oscillating with a coil inbetween. Along with the base design of the $\mathrm{KEH}$, there are numerous factors that must be simultaneously optimized; coil gauge, coil inner and outer diameter, coil thickness, spring frequency, and magnet separation. Optimizations of these parameters are simulated using electromagnetic and solid model analysis, and verified through construction and testing of the devices.

\section{DEVICE DESIGN}

The KEH device schematic is shown in Figure 1. The two magnets are rigidly attached together with like poles facing each other. This condenses the magnetic field lines such that there is a large magnetic field gradient between the magnets. A coil rests between the two magnets such that any small displacement will cause the coil to travel through a large magnetic field gradient. With this system either the magnets or the coil can be the oscillating spring mass system, while the other is stationary.
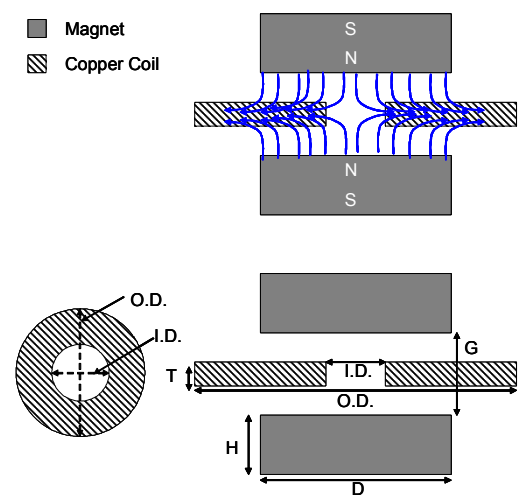

Figure 1. (Top) EH design with magnetic flux lines. (Bottom) EH dimensions; O.D. = coil outer diameter, I.D. = coil inner diameter, $\mathrm{T}=$ coil thickness, $\mathrm{H}=$ magnet thickness, $\mathrm{G}=$ magnet separation, $\mathrm{D}=$ magnet diameter

The coil is made of copper magnet wire with bondable and insulator layers. The magnets are rare earth magnets made of neodymium, more specifically, our designs use N52. The springs on the KEH, as can be seen in Figure 2, are chemically etched T301 Stainless Steel Full Hard Tempered. The upper 
and lower springs are attached via screws to the surrounding support structure so that the springs can be replaced easily for the purposes of modifying the resonant frequency.

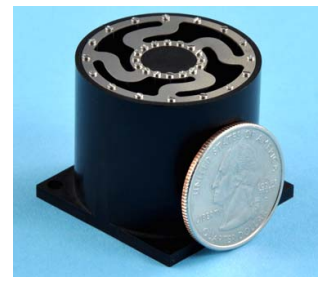

Figure 2. SSC PACIFIC KEH device, mass $=36.5$ grams, volume $=27 \mathrm{~cm}^{3}$. The metal springs are visible on top

\section{DESIGN VARIATIONS OVERVIEW}

The configuration of the magnets, coupled with the placement of the coil is the feature that allows for small oscillations to produce useable power. With the assumption that a mass and/or volume constraint has been set by the system level application, the magnet size can be chosen. The magnet thickness $\mathrm{H}$, as shown in Figure 1, does not increase the field strength or the magnetic flux as significantly as increasing the area of the magnet. The closer together the magnets are spaced, the sharper the flux line gradient between the magnets becomes, and the extractable power increases; this has been verified in Ansoft Maxwell 2D and 3D simulations, Figure 3, and experimentally. The increased magnetic flux gradient increases the energy density stored between the magnets which thereby increases the ability to extract energy for low amplitude vibrations. The final separation of the magnets is dictated by the frequency of the vibration in the application environment and therefore the amount of mass-spring displacement required. Within an environment, the resonant frequencies in which peak accelerations occur are the design emphasis for the $\mathrm{KEH}$ mass-spring system. The mass-spring system is designed to have a resonant frequency that matches the frequency in the environment spectra that has the most potential power. This is shown in Figure 4 along with the generated voltage output.

\section{Power verse Magnet Spacing at $10 \mathrm{mg}$ and $44 \mathrm{~Hz}$}

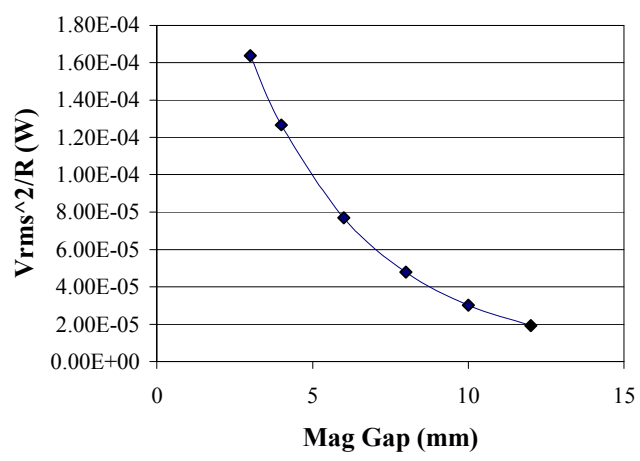

Figure 3. Ansoft Maxwell 2D simulation of open circuit power from an energy harvester coil as a function of the spacing between the magnets. The coil size was fixed. Note: lab results are consistently higher than simulations
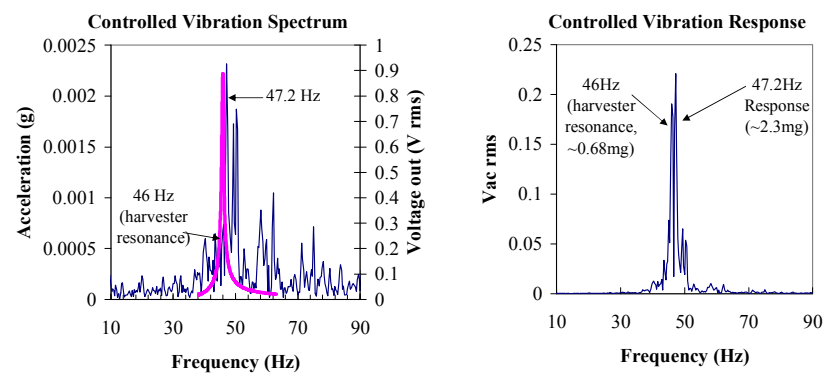

Figure 4. (Left) Frequency profile (light gray, axis on right) of $\mathrm{KEH}, 27 \mathrm{~cm}^{3}$ volume and 36.5 grams mass, laid over natural environment vibration (dark, axis on left). (Right) Output voltage $\mathrm{AC}$ from energy harvester. As seen in the right image, the environmental peak at the $\mathrm{KEH}$ resonance is amplified, and the large off resonance peak is still extracted by the KEH

After the mass-spring system is designed, the coil needs to be optimized. This is done using MatLab simulations and Ansoft Maxwell 2D and 3D. To maximize the useable power extracted from the coil, there is a balance between voltage induced, coil resistance and load resistance. For example, as the gauge of the wire increases (conductor diameter decreases) the resistance increases, but the number of turns increases which increases the voltage. This does not directly mean more power will be delivered to the load if the load resistance is kept the same. The coil resistance and number of turns, assuming hexagonal packing, can be calculated if the coil dimensions, nominal wire and conductor size are known. The resistance and number of turns in the coil are then entered into Maxwell for the electro-magnetic coupling simulation. Similarly, with a larger coil volume, there is an increase in resistance, but again, also greater voltage. Due to the nonlinearity of the magnetic flux lines, there is an optimum volume in which the coil can extract the maximum amount of power. To find this volume, Ansoft Maxwell was used to simulate various volumes, as shown in Figure 6.

Another factor that needs to be considered is the damping within the system. There are two types of damping present, mechanical and electrical. The mechanical damping is due to the spring losses and the airflow squeeze film damping between the magnet and the coil. The electrical damping occurs when a load is attached across the harvester. There is a change in total damping when the circuit is loaded because at this point there is a current draw from the coil, which causes a magnetic field. This induced magnetic field opposes the magnetic field from the moving magnets causing a damping of their motion.

\section{Simulations}

The objective of this work is to develop the smallest volume KEH capable of powering a wireless node using exclusively ambient environment energy. To do this there are numerous factors, all of which are dependent variables, which are dictated by the environmental vibration source.

The displacement of the mass-spring system is determined by the acceleration and the frequency at which the KEH operates. The displacement can be calculated by solving the equation of motion [6]. As shown in Figure 3, the separation 
of the magnets influences the power available in the coil. The closer the magnets are together the larger the magnetic flux coupling within the coil. While a thin coil allows for closely spaced magnets, the coil thickness has to be large enough to extract enough voltage to overcome diode drops in the rectifier. This introduces a cyclic relationship between the coil thickness and the magnet separation. The more coil turns between the magnets, the larger the voltage, but also the larger the resistance and the larger the separation of the magnets. As the magnets separate the magnetic flux gradient decreases causing less induced voltage.

The inner and outer diameters of the coil are constrained by the coil resistance versus the voltage desired. The total coil resistance increases as the outer radius squared. This was found setting up a piecewise equation to account for each individual wire within a given volume. An example is plotted in Figure 5, which shows the squared relationship, where the coefficient in front represents a factor determined by the coil thickness, the resistivity of the conductor, and other geometric parameters.

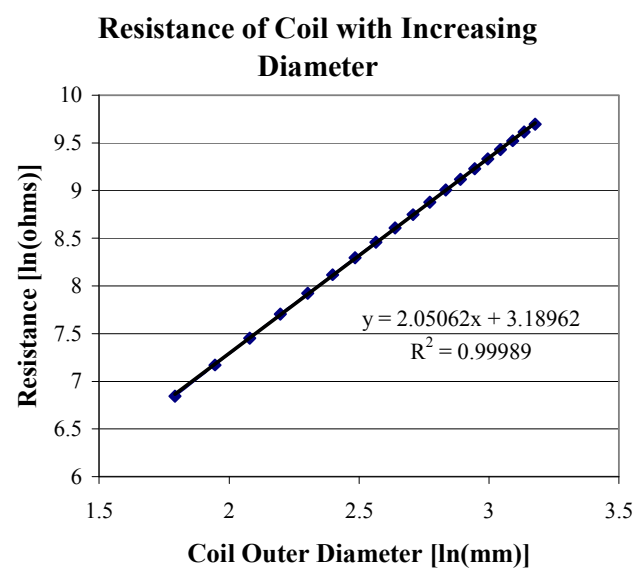

Figure 5. The computed resistance of a copper coil (50AWG) with a fixed inner diameter and a varying outer diameter

Simulations in Ansoft Maxwell 2D are run to determine at which point the resistance added is too costly compared to the voltage gained, or makes the matching resistance too large for the application. For small magnets the resistance added as the inner diameter decreases is minimal compared to the voltage gained, and can be minimized as much as possible. For larger size magnets, the inner diameter must be increased to lower resistance, and the outer diameter can be increased until there is no benefit, to allow for reasonable load matching. The outer coil diameter is also constrained by the desired physical size of the $\mathrm{KEH}$ packaging. Simulation results for a $50.8 \mathrm{~mm}$ diameter, $3.175 \mathrm{~mm}$ thick magnet with various coil OD and ID is plotted in Figure 6.

Through Maxwell 2D simulations it has been found that the most power can be extracted around the edge of the magnet, Figure 7. The plot shown is the power available measured within a coil band with a constant radial width of 1 $\mathrm{mm}$ with varying placements of $0.5 \mathrm{~mm}$ steps. This result is consistent with the concentration of magnetic flux lines and increased magnetic strength around the edges of a magnet.
This principle is shown in Figure 8, where the field strength was measured on a N52 magnet of diameter $15.875 \mathrm{~mm}$ using a SENIS Single Axis Magnetic Field Transducer YM 12-3-5$5 \mathrm{~T}$.

Peak Power vs. Inner Diameters

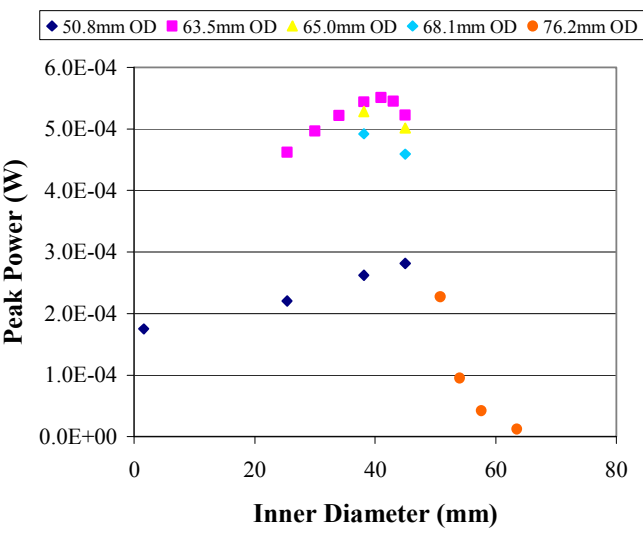

Figure 6. Simulated peak power output vs. inner diameter of coil, grouped by outer diameter, for an acceleration of $10 \mathrm{mg}$ rms. The thickness of coil was $2 \mathrm{~mm}$; volumes were constrained by a coil resistance $<225 \mathrm{k} \Omega$ at $43.8 \mathrm{~Hz}$

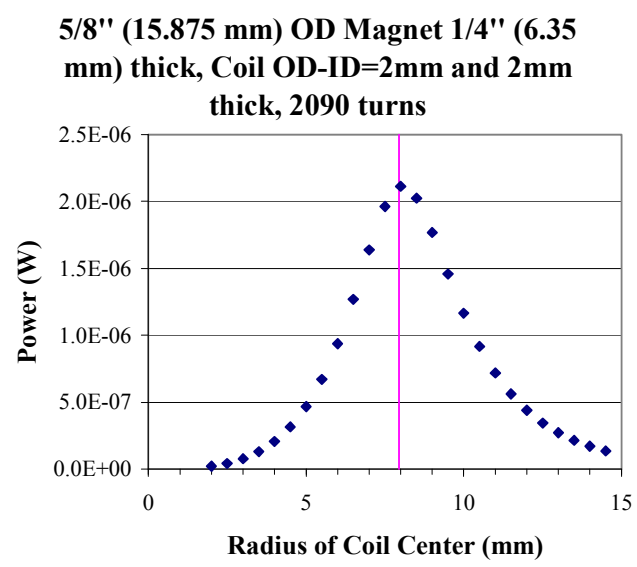

Figure 7. Ansoft Maxwell 2D simulation results of a power sweep using a constant width inductor across the radial axis of the magnets, the vertical line is the edge of the magnet.

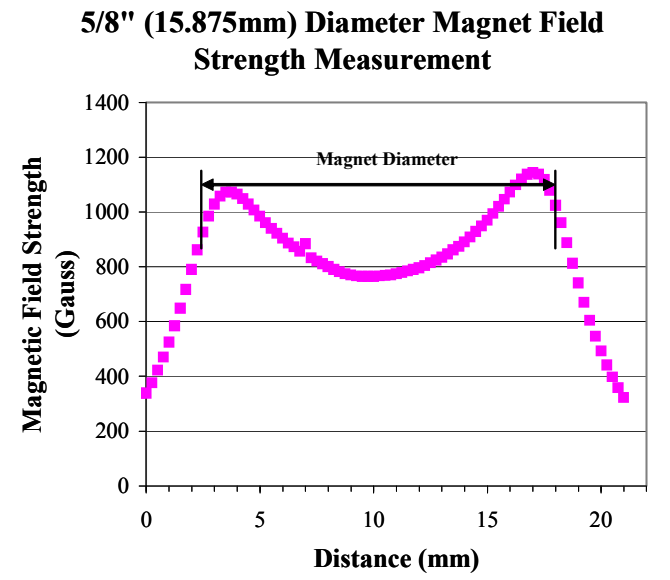

Figure 8. Measured magnetic field strength across the diameter of a $15.875 \mathrm{~mm}$ diameter $\mathrm{N} 52$ magnet with a $6.35 \mathrm{~mm}$ thickness 
Because the coil size is roughly known based upon the size of the magnet, a preliminary coil size is chosen prior to the Maxwell 2D simulations. Guessing the coil size is used to determine the wire gauge that would be best suited. The larger the coil gauge, the small diameter the wire. The smaller the wire diameter, the larger the wire resistance per length; in addition due to the reduced wire diameter more turns can fit into a given volume. The higher number of turns leads to a higher output voltage. Still, there is one more caveat, the higher the gauge the smaller the conductor to insulator and bondable coat ratio. This means, though, more turns would exist in a coil with a higher gauge; there would be less conductor volume and more volume occupied by insulator and bondable material. This effect varies based upon the specifications given by the wire manufacturer. After the simulations are run to find the coil dimensions, the coil gauge calculation is repeated to ensure the selected coil gauge remains valid.

Another parameter that must be estimated prior to simulation is the damping coefficient. There are two types of damping in our system, mechanical and electrical. From previously measured open circuit displacements, the mechanical damping ratio is approximated as $1 \%$. For open circuit calculations, only the mechanical damping is present. When a load is added, then the current draw causes the electrical damping. The maximum power can be extracted when the electrical damping and the mechanical damping are equal [7]. Therefore, for loaded circuit simulations, the load resistance is varied until a max power to the load is found. To find the actual damping, the KEH is physically built and then tested at the simulation frequency and amplitude. The open circuit displacement is measured with a Microtrak II displacement meter and compared to the equation of motion to find the mechanical damping. Also by varying the load resistance, the maximum power to the load can be found, as shown in Figure 9. Knowing the displacement at the peak power, the total damping can be calculated, and compared to the mechanical damping. It is possible that the electrical damping is too high for a given load resistance and in this case the electrical damping can be lowered by increasing the load resistance, increasing the magnet spacing, changing the coil OD and ID and/or adding mass. This is due to the high coupling between the coil and magnet.

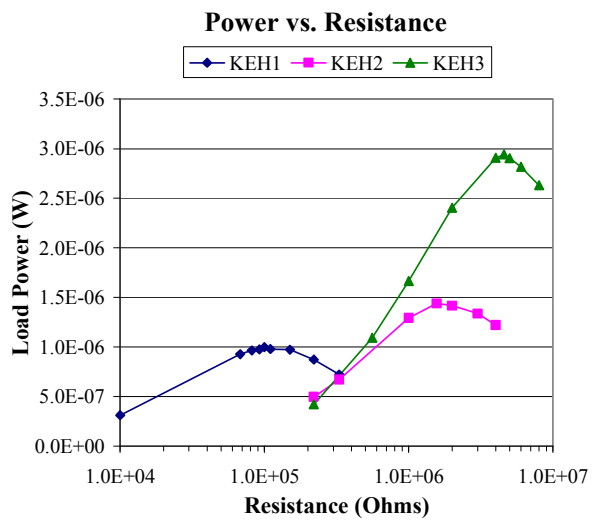

Figure 9. Measured load power vs. matching resistance for three different KEHs

\section{CONCLUSION}

The process for designing a $\mathrm{KEH}$ is a process that is dictated by numerous non-linear coupled parameters. This leads to a method that relies on continually changing the variables in a cyclic fashion. The design process described is based solely on SSC PACIFIC's device. There are other electromagnetic $\mathrm{KEH}$ devices, [8], that use environment vibrations with different core designs. In addition, there are electromagnetic $\mathrm{KEH}$ that rely on a single large impulse of energy to create power [9] rather than a continual vibration source. Furthermore, there are $\mathrm{KEH}$ that use piezoelectric materials [10] for the conversion of kinetic energy into electrical energy. All of these varying harvesters have similarities, but simultaneously pose varying complex design issues.

\section{REFERENCES}

[1] P.D Mitcheson, G.K Rao, T.C. Green, "Energy Harvesting From Human and Machine Motion." Proceedings of the IEEE. Vol. 96, No. 9, September 2008

[2] D.P. Arnold. "Review of Microscale Magnetic Power Generation." IEEE Transactions on Magnetics, VOL. 43, NO. 11, Nov. 2007 39403951

[3] S.P. Beeby, "A micro electromagnetic generator for vibration energy harvesting. " J Micromech. Microeng. 17 (2007) 1257-1265

[4] S. Roundy, "On the Effectiveness of Vibration-based Energy Harvesting" JIMSS. Vol. 16 Oct 05. 809-823

[5] B.R. Hunt, P.M. Sullivan, R.L. Waters, "Micro-electro-mechanical system (MEMS) and apparatus for generating power responsive to mechanical vibration" U.S. Patent Number 7,501,726. 10 March 2009

[6] T. Sterken, K. Baert, C. Van Hoof, R. Puers, G. Borghs, P.Fiorini. "Comparative Modelling for Vibration Scavengers." IEEE (2004) $1249-1252$

[7] S.P. Beeby, M.J. Tudor, N.M. White, "Energy harvesting vibration sources for microsystems applications.” Meas. Sci. Technol. 17 (2006) R175-R195

[8] C.R. Saha, T. O’Donnell, N.Wang, P. McCloskey. "Electromagnetic generator for harvesting energy from human motion." Sensors and Actuators A 147 (2008) 248-253

[9] EnOcean Energy Harvesting Devices. EnOcean (C) 2009. http://www.enocean.com/en/energy-harvesting/

[10] J.A. Paradiso, T. Starner. "Energy Scavenging for Mobile and Wireless Electronics.” IEEE ComSoc. 1536-1268/05

[11] S.P. Beeby, "Macro and Micro Scale Electromagnetic Kinetic Energy Harvesting Generators.” DTIP 2006 